\title{
Four year efficacy of prophylactic human papillomavirus quadrivalent vaccine against low grade cervical, vulvar, and vaginal intraepithelial neoplasia and anogenital warts: randomised controlled trial
}

\author{
The FUTURE I/II Study Group
}

\section{Correspondence to: J Dillner,} Department of Medical Microbiology, Lund University, Malmö University Hospital, SE20502 Malmö, Sweden joakim.dillner@med.lu.se

Cite this as: BMJ 2010;340:c3493 doi:10.1136/bmj.c3493

\section{ABSTRACT}

Objectives To evaluate the prophylactic efficacy of the human papillomavirus (HPV) quadrivalent vaccine in preventing low grade cervical, vulvar, and vaginal intraepithelial neoplasias and anogenital warts (condyloma acuminata).

Design Data from two international, double blind, placebo controlled, randomised efficacy trials of quadrivalent HPV vaccine (protocol 013 (FUTURE I) and protocol 015 (FUTURE II)). The trials were to be 4 years in length, and the results reported are from final study data of 42 months' follow-up.

Setting Primary care centres and university or hospital associated health centres in 24 countries and territories around the world.

Participants 17622 women aged $16-26$ years enrolled between December 2001 and May 2003. Major exclusion criteria were lifetime number of sexual partners (>4), history of abnormal cervical smear test results, and pregnancy.

Intervention Three doses of quadrivalent HPV vaccine (for serotypes $6,11,16$, and 18 ) or placebo at day 1 , month 2 , and month 6.

Main outcome measures Vaccine efficacy against cervical, vulvar, and vaginal intraepithelial neoplasia grade I and condyloma in a per protocol susceptible population that included subjects who received all three vaccine doses, tested negative for the relevant vaccine HPV types at day 1 and remained negative through month 7 , and had no major protocol violations. Intention to treat, generally HPV naive, and unrestricted susceptible populations were also studied.

Results In the per protocol susceptible population, vaccine efficacy against lesions related to the HPV types in the vaccine was $96 \%$ for cervical intraepithelial neoplasia grade I (95\% confidence interval 91\% to 98\%), $100 \%$ for both vulvar and vaginal intraepithelial neoplasia grade I ( $95 \%$ Cls $74 \%$ to $100 \%, 64 \%$ to $100 \%$ respectively), and $99 \%$ for condyloma (96\% to $100 \%$ ). Vaccine efficacy against any lesion (regardless of HPV type) in the generally naive population was 30\% (17\% to $41 \%$ ), $75 \%$ ( $22 \%$ to $94 \%$ ), and $48 \%$ (10\% to $71 \%$ ) for cervical, vulvar, and vaginal intraepithelial neoplasia grade I, respectively, and $83 \%$ (74\% to $89 \%$ ) for condyloma.

Conclusions Quadrivalent HPV vaccine provided sustained protection against low grade lesions attributable to vaccine HPV types $(6,11,16$, and 18$)$ and a substantial reduction in the burden of these diseases through 42 months of follow-up.

Trial registrations NCT00092521 and NCT00092534.

\section{INTRODUCTION}

Human papillomaviruses (HPVs) are responsible for about 500000 cases of cervical cancer a year globally ${ }^{1}$ and 10 million further cases of high grade cervical intraepithelial neoplasias (grades II or III), ${ }^{2}$ immediate precursors to malignancy. It is estimated that 30 million women and men acquire anogenital warts (condyloma acuminata) or low grade cervical intraepithelial neoplasia each year, ${ }^{2}$ which may be an underestimation given the inadequacy of reporting in many countries and evidence of a rising incidence over time. Although many low grade lesions of the lower genital tract resolve spontaneously in immunocompetent subjects, this type of lesion contributes greatly to the clinical and economic burden of HPV disease in women. The psychosocial ${ }^{34}$ and economic ${ }^{56}$ implications of condyloma are substantial and reflect, in part, the high transmission rates and inadequacy of available treatment options in achieving prolonged response rates. ${ }^{7-9}$ Cervical intraepithelial neoplasia grade I can contain a variety of low or high risk HPV types, ${ }^{10-12}$ whereas anogenital warts are (in up to $90 \%$ of cases) caused by either of two low risk HPV types-namely, 6 and 11.91314

The quadrivalent HPV vaccine (for types 6, 11, 16, and 18) has the potential to prevent about $70 \%$ of cervical cancers ${ }^{15}$ and $90 \%$ of condyloma ${ }^{913}$ by targeting HPV types 16 and 18 and types 6 and 11, respectively. Clinical trials have shown that in the per protocol population (that is, subjects naive to a given HPV type(s) at baseline and throughout the three dose vaccination) vaccine efficacy against cervical intraepithelial neoplasia grade II-III or adenocarcinoma in situ was $99 \%$ (95\% confidence interval 93\% to 
$100 \%) .{ }^{16}$ Efficacy against vulvar and vaginal intraepithelial neoplasia grade II-III was $100 \%(72 \%$ to $100 \%) .{ }^{17}$ High efficacy against condyloma has also been demonstrated $(100 \%(92 \%$ to $100 \%)) .{ }^{18}$ These data have led to regulatory approval of the vaccine in roughly 100 countries for the prevention of cervical cancer, cervical cancer precursor lesions, and condyloma in girls and women aged 9-26 years. In some countries, the approved indication also includes vulvar and vaginal cancers.

Details of different patient populations analysed for efficacy of quadrivalent human papillomavirus (HPV) vaccine

Per protocol susceptible population

- Subjects

Received all three vaccination doses

Were negative for HPV types 6, 11, 16, and 18 at day 1 (by serology and polymerase chain reaction (PCR)) and through month 7 (by PCR)

Generally did not deviate from study protocol

- Case counting began after month 7

- Represents an ideal population under ideal study conditions and approximates to a sexually naive population

- Used for analyses of disease related to HPV types 6, 11, 16, and 18

Unrestricted susceptible population

- Subjects

Received $\geq 1$ vaccination dose

Were negative for HPV types $6,11,16$, and 18 at day 1 (by serology and PCR)

Had any follow-up visit

- Case counting began after day 1

- Represents a broader population than per protocol susceptible population, including subjects completing the full three vaccination doses and others who received only one or two doses

- Used for analyses of disease related to HPV types 6, 11, 16, and 18

Generally HPV naive population

- Subjects

Received $\geq 1$ vaccination

Were negative for HPV types $6,11,16$, and 18 at day 1 (by serology and PCR), negative at day 1 for other high risk HPV types with PCR assays available $(31,33,35,39,45,51$, $52,56,58$, and 59), and had a negative cervical smear test result on day 1 Had any follow-up visit

- Case counting began after day 1

- Represents an approximation of a "real world" population of HPV naive women

- Used for analyses of disease due to any HPV type

Intention to treat population

- Subjects

Received $\geq 1$ vaccination

Had any follow-up visit ( $>98 \%$ of all subjects enrolled)

- Case counting began after day 1

- Represents a population of women with past and current HPV exposures as well as those presumably naive to HPV

- Used for analyses of disease related to

HPV types 6, 11, 16, and 18

Any HPV type
The contribution of HPV types $6,11,16$, and 18 to low grade neoplasias has not been well elucidated. The HPV types have been found in $25-50 \%$ of low grade cervical and vulvovaginal neoplasias, ${ }^{10-12}$ but assigning causality is difficult because most of these lesions contain multiple HPV types. Whether elimination of some of the HPV types in a multiple infection will prevent disease can be proved only through vaccination. This report represents a combined analysis of quadrivalent HPV vaccine protocols 013 (FUTURE I trial) and 015 (FUTURE II trial), focusing on the efficacy of the vaccine in preventing low grade cervical and vulvovaginal lesions (grade I neoplasias and condyloma) after an average of 42 months of follow-up. We also sought to describe the proportion of the low grade disease burden that can be prevented by vaccination against HPV types $6,11,16$, and 18 .

\section{METHODS}

\section{Study designs}

Data are considered from two international, double blind, placebo controlled, randomised efficacy trials of the quadrivalent HPV vaccine (protocol $013^{18}$ (FUTURE I, NCT00092521) and protocol 015 ${ }^{19}$ (FUTURE II, NCT00092534)). These trials were similar in design and infrastructure and were conducted among women aged 16-26 years from North America, Latin America, Europe, and Asia Pacific. Primary efficacy end points assessed in protocol 013 included $(a)$ condyloma, vulvar and vaginal intraepithelial neoplasia, or vulvar and vaginal cancer related to HPV types $6,11,16$, or 18; and $(b)$ cervical intraepithelial neoplasia, adenocarcinoma in situ, or cervical cancer related to HPV types $6,11,16$, or 18 . The primary efficacy end points assessed in protocol 015 were cervical intraepithelial neoplasia grades II-III and cervical cancer related to HPV types 16 or 18 .

Pregnant women and those with a history of $>4$ lifetime sexual partners or history of an abnormal cervical smear test result were not eligible to participate in these trials. The institutional review board at each participating centre approved the protocol, and informed consent was obtained from all participants. The current report details the complete follow-up data from protocols 013 and 015 , representing a mean follow-up period of 42 months.

\section{Vaccine}

In each of the studies, eligible subjects were randomised in a 1:1 ratio to receive three doses of the quadrivalent (HPV types 6, 11, 16, and 18) LI virus-like particle vaccine (Gardasil, Merck, Whitehouse Station, NJ, USA) or placebo at day 1 , month 2 , and month 6 (additional vaccination regimens included as part of protocol $013 \mathrm{did}$ not contribute to the data reported here).

\section{Study procedures}

Detailed cervicovaginal examinations were performed at the scheduled day 1 and month 7 visits, including cervical collections for cervical smear testing (ThinPrep, Cytyc Corporation, Boxborough, MA, USA), 
Table 1|Efficacy of quadrivalent human papillomavirus (HPV) vaccine against low grade lesions attributable to vaccine HPV types (6, 11, 16, and 18 ): analysis of per protocol susceptible population*

\begin{tabular}{|c|c|c|c|c|c|c|}
\hline \multirow[b]{2}{*}{ Lesion and related HPV type $†$} & \multicolumn{2}{|c|}{ Vaccine group } & \multicolumn{2}{|c|}{ Placebo group } & \multicolumn{2}{|c|}{ Vaccine efficacy $(\%(95 \% \mathrm{Cl}))$} \\
\hline & $\begin{array}{l}\text { No of cases/ } \\
\text { No of subjects }\end{array}$ & $\begin{array}{c}\text { Person } \\
\text { years at risk }\end{array}$ & $\begin{array}{l}\text { No of cases/ } \\
\text { No of subjects }\end{array}$ & $\begin{array}{c}\text { Person } \\
\text { years at risk }\end{array}$ & Unadjusted & Adjusted $\ddagger$ \\
\hline Cervical intraepithelial neoplasia grade I (HPV 6, 11, 16, 18): & $7 / 7629$ & 22456.6 & $168 / 7632$ & 22306.3 & $95.9(91.3$ to 98.4$)$ & 95.9 (91.2 to 98.1$)$ \\
\hline HPV 6 or 11 & $0 / 6688$ & 19756.5 & $45 / 6619$ & 19452.6 & $100(91.6$ to 100$)$ & $100(\mathrm{NA})$ \\
\hline HPV 16 & $6 / 6448$ & 19122.2 & $97 / 6257$ & 18531.1 & $94.0(86.5$ to 97.9$)$ & $93.9(86.2$ to 97.3$)$ \\
\hline HPV 18 & $1 / 7158$ & 21118.9 & 47/7092 & 20827.4 & $97.9(87.7$ to 99.9$)$ & 97.9 (84.8 to 99.7$)$ \\
\hline Vulvar intraepithelial neoplasia grade I (HPV 6, 11, 16, 18): & $0 / 7665$ & 23042.9 & $16 / 7669$ & 23041.1 & $100(74.1$ to 100$)$ & $100(\mathrm{NA})$ \\
\hline HPV 6 or 11 & $0 / 6718$ & 20179.0 & $16 / 6647$ & 19982.8 & $100(74.3$ to 100$)$ & $100(\mathrm{NA})$ \\
\hline HPV 16 & $0 / 6455$ & 19417.0 & $0 / 6269$ & 18859.6 & NA & NA \\
\hline HPV 18 & $0 / 7190$ & 21613.9 & $0 / 7119$ & 21418.9 & NA & NA \\
\hline Vaginal intraepithelial neoplasia grade I (HPV 6, 11, 16, 18): & $0 / 7665$ & 23042.9 & $12 / 7669$ & 23049.8 & $100(64.0$ to 100$)$ & $100(\mathrm{NA})$ \\
\hline HPV 6 or 11 & $0 / 6718$ & 20179.0 & $6 / 6647$ & 19999.0 & $100(15.8$ to 100$)$ & $100(\mathrm{NA})$ \\
\hline HPV 16 & $0 / 6455$ & 19417.0 & $7 / 6269$ & 18859.6 & $100(32.6$ to 100$)$ & $100(\mathrm{NA})$ \\
\hline HPV 18 & $0 / 7190$ & 21613.9 & $2 / 7119$ & 21418.9 & $100(<0$ to 100$)$ & $100(\mathrm{NA})$ \\
\hline Condyloma (HPV 6, 11, 16, 18): & $2 / 7665$ & 23039.6 & $190 / 7669$ & 22849.4 & 99.0 (96.2 to 99.9) & 99.0 (95.8 to 99.7) \\
\hline HPV 6 or 11 & $2 / 6718$ & 20175.7 & $186 / 6647$ & 19798.0 & 98.9 (96.1 to 99.9) & 98.9 (95.7 to 99.7) \\
\hline HPV 16 & $0 / 6455$ & 19417.0 & $23 / 6269$ & 18839.4 & $100(83.1$ to 100$)$ & $100(\mathrm{NA})$ \\
\hline HPV 18 & $0 / 7190$ & 21613.9 & $11 / 7119$ & 21406.8 & $100(60.5$ to 100$)$ & $100(\mathrm{NA})$ \\
\hline
\end{tabular}

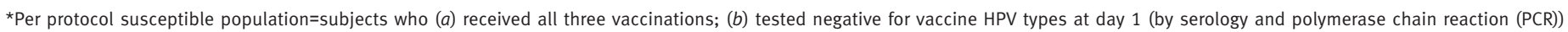
and through month 7 (by PCR); and (c) generally did not deviate from the protocol. Case counting began after month 7.

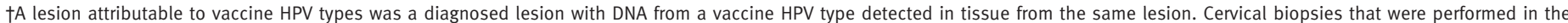
absence of an abnormal cervical smear test result at the antecedent visit were excluded.

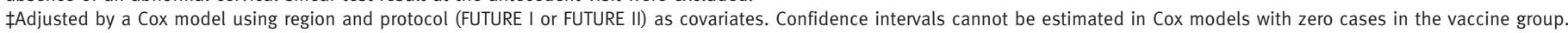

comprehensive anogenital inspection, and a series of cervical or anogenital swab collections (that is, endocervical or ectocervical, combined labial-vulvar-perineal, and perianal swabs) for HPV DNA testing. Protocol 013 had an additional scheduled visit at month 3, during which gynaecological examination and cervical or anogenital swab collection occurred (but not serum sampling or cervical smear testing). Cytology specimens were evaluated using the 2001 Bethesda system. ${ }^{20}$ Colposcopy referral was based on a decision algorithm. Biopsy material was first read for clinical management by pathologists at a central laboratory (Diagnostic Cytology Laboratories, Indianapolis, IN) and then read for end point determination by a blinded panel of four pathologists.

After the three dose vaccination, protocol 013 participants were to return for follow-up assessments every six months (until month 48), whereas protocol 015 participants were seen every 12 months (until month 48). Interim visits were required six months after detection of atypical squamous cells of undetermined significance or low grade squamous intraepithelial lesions to provide the opportunity for repeat cervical smear testing and, if indicated, further colposcopic evaluation and biopsy. If an anogenital lesion was detected, investigators were instructed to obtain specimens representing each affected area and each morphology in a given area. Definitive treatment was based on local standards of care.

Sensitive and specific multiplex polymerase chain reaction assays were used for HPV typing of biopsy samples for HPV 6, 11, 16, 18, and 10 other HPV types (31, $33,35,39,45,51,52,56,58$, and 59$)$. For the low grade analyses reported here, a case required a consensus diagnosis from the pathology panel of cervical, vaginal, or vulvar intraepithelial neoplasia grade I, or condyloma with DNA of HPV types $6,11,16$, or 18 detected in an adjacent section from the same tissue block.

In 2005 the International Society for the Study of Vulvovaginal Disease changed nomenclature for vulvar intraepithelial neoplasia and categorised it as usual (u-VIN, HPV related) or differentiated (d-VIN, not HPV related) types. The term VIN I was abandoned, and terms VIN II and VIN III were merged. ${ }^{21}$ In this report, however, we have maintained the original nomenclature (VIN I) that was used by the pathology panel during the course of the studies.

\section{Statistical methods}

The box lists the criteria and rationale for the analysis populations considered in this report (per protocol susceptible, intention to treat, generally HPV naive, and unrestricted susceptible). The statistical analysis plan specified that determination of the efficacy of the vaccine was to be based on analyses of the per protocol susceptible population; other populations were analysed only for supportive results.

Vaccine efficacy analyses were performed based on low grade lesion type, pooling subjects across the studies by vaccination group (vaccine or placebo). Data were analysed to determine vaccine efficacy against lesions attributable to vaccine HPV types $(6,11,16$, and 18) as well as to any tested HPV type, with the latter analyses including the 10 non-vaccine HPV types for which polymerase chain reaction testing was performed in protocols 013 and 015 (types 31, 33, 35, 
Table 2 |Efficacy of quadrivalent human papillomavirus (HPV) vaccine against low grade lesions attributable to vaccine HPV types (6, 11, 16, and 18$)$ : analysis of unrestricted susceptible population*

\begin{tabular}{|c|c|c|c|c|c|c|}
\hline \multirow[b]{2}{*}{ Lesion and related HPV type $†$} & \multicolumn{2}{|c|}{ Vaccine group } & \multicolumn{2}{|c|}{ Placebo group } & \multicolumn{2}{|c|}{ Vaccine efficacy $(\%(95 \% \mathrm{Cl}))$} \\
\hline & $\begin{array}{l}\text { No of cases/ } \\
\text { No of subjects }\end{array}$ & $\begin{array}{c}\text { Person } \\
\text { years at risk }\end{array}$ & $\begin{array}{l}\text { No of cases/ } \\
\text { No of subjects }\end{array}$ & $\begin{array}{c}\text { Person } \\
\text { years at risk }\end{array}$ & Unadjusted & Adjusted $\ddagger$ \\
\hline Cervical intraepithelial neoplasia grade I (HPV 6, 11, 16, 18): & $12 / 8375$ & 29081.4 & $235 / 8430$ & 29063.8 & 94.9 (90.9 to 97.4$)$ & 94.9 (90.8 to 97.1$)$ \\
\hline HPV 6 or 11 & $2 / 7420$ & 25879.5 & $60 / 7466$ & 25870.6 & 96.7 (87.4 to 99.6$)$ & $96.6(86.2$ to 99.2$)$ \\
\hline HPV 16 & $6 / 7113$ & 24979.5 & $137 / 7141$ & 24944.4 & $95.6(90.2$ to 98.4$)$ & 95.6 (90.1 to 98.1$)$ \\
\hline HPV 18 & 4/7906 & 27537.1 & $66 / 7970$ & 27622.0 & 93.9 (83.7 to 98.4$)$ & 93.9 (83.4 to 97.8$)$ \\
\hline Vulvar intraepithelial neoplasia grade I (HPV 6, 11, 16, 18): & $2 / 8497$ & 29825.1 & $20 / 8532$ & 29995.6 & 89.9 (58.6 to 98.9$)$ & $89.9(57.0$ to 97.6$)$ \\
\hline HPV 6 or 11 & $2 / 7529$ & 26429.1 & $18 / 7553$ & 26582.1 & 88.8 (53.3 to 98.7$)$ & 88.8 (51.7 to 97.4$)$ \\
\hline HPV 16 & $0 / 7220$ & 25363.4 & $2 / 7222$ & 25444.7 & 100 ( $(0$ to 100$)$ & $100(\mathrm{NA})$ \\
\hline HPV 18 & $0 / 8022$ & 28179.3 & $0 / 8067$ & 28387.4 & NA & NA \\
\hline Vaginal intraepithelial neoplasia grade I (HPV 6, 11, 16, 18): & $0 / 8497$ & 29829.3 & $18 / 8532$ & 30000.6 & $100(77.1$ to 100$)$ & $100(\mathrm{NA})$ \\
\hline HPV 6 or 11 & $0 / 7529$ & 26433.3 & $7 / 7553$ & 26600.8 & $100(30.2$ to 100$)$ & $100(\mathrm{NA})$ \\
\hline HPV 16 & $0 / 7220$ & 25363.4 & $10 / 7222$ & 25436.7 & $100(55.3$ to 100$)$ & $100(\mathrm{NA})$ \\
\hline HPV 18 & $0 / 8022$ & 28179.3 & $5 / 8067$ & 28387.4 & $100(<0$ to 100$)$ & 100 (NA) \\
\hline Condyloma (HPV 6, 11, 16, 18): & $10 / 8497$ & 29807.5 & $249 / 8532$ & 29692.7 & $96.0(92.5$ to 98.1$)$ & $96.0 \S(92.5$ to 97.9$)$ \\
\hline HPV 6 or 11 & $9 / 7529$ & 26414.0 & $243 / 7553$ & 26287.7 & 96.3 (92.9 to 98.3) & 96.3 (92.8 to 98.1) \\
\hline HPV 16 & $1 / 7220$ & 25360.9 & $27 / 7222$ & 25411.6 & 96.3 (77.5 to 99.9$)$ & 96.2 (72.3 to 99.5$)$ \\
\hline HPV 18 & $0 / 8022$ & 28179.3 & $19 / 8067$ & 28364.7 & $100(78.4$ to 100$)$ & $100(\mathrm{NA})$ \\
\hline
\end{tabular}

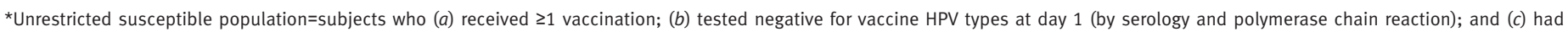
any follow-up visit. Case counting began after day 1.

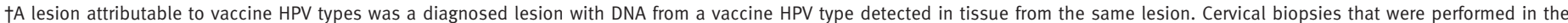
absence of an abnormal cervical smear test result at the antecedent visit were excluded.

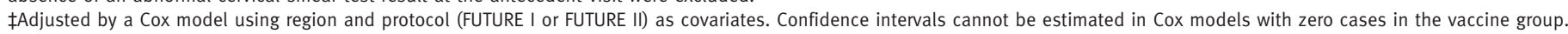
§Proportional hazard assumption was violated for the treatment effect. The vaccine efficacy was reported as average effect.

$39,45,51,52,56,58$, and 59). Unadjusted vaccine efficacy rates were calculated as $(1-$ relative risk $) \times 100$, with corresponding 95\% confidence intervals estimated via an exact conditional procedure. ${ }^{22}$ Relative risk was defined as the ratio of the incidence rate in the vaccine group divided by the incidence rate in the placebo group, using person-time incidence rates.

In order to adjust for study effect and country effect, vaccine efficacy rates were also calculated using a Cox model in which protocol and region were included as cofactors. Regarding the method used for adjusting for study effect and region, we settled for using a Cox regression model because it is more distribution-free (semi-parametric) than Poisson regression. When calculating the vaccine efficacy as 1-relative risk (that is, 1-hazard ratio, with the Cox model), the only assumption used was that the ratio of hazards is constant over time (but the underlying risks can vary over time). An alternative analysis with Poisson regression (using the PROC GENMOD procedure in the SAS statistical package) gave almost identical results (supplementary table A on bmj.com).

Many of the efficacy end points analysed here and in previous reports are composite, capturing more than one pathological diagnosis or more than one HPV type, or both. Subjects were counted as a single case for a composite end point regardless of whether they met the criteria for only one or for more of its components; however, individual subjects were counted within each of the individual components for which the criteria were met. For example, a subject with both HPV type 6 and type 16 identified in an emergent anogenital wart was counted once within each HPV-specific analysis but only once in the composite end point of condyloma related to HPV types 6, 11, 16, or 18 .

\section{RESULTS}

\section{Subject population}

A total of 17599 women aged 16-26 years were randomised and received one or more dose of the quadrivalent HPV vaccine or placebo. Baseline characteristics for the randomised population, which were similar in the vaccine and placebo groups, have been published previously. ${ }^{23}$ Overall, mean age at enrolment was 20.0 years, mean age at first sexual intercourse was 16.7 years, non-virgins had a mean of 2.1 lifetime sexual partners, and $11.2 \%$ of subjects $(1955 / 17433)$ had abnormal cervical cytology at enrolment. The day 1 prevalence for one or more of the HPV types included in the vaccine was $14.7 \%$ by polymerase chain reaction (2593/17622) and 19.8\% by serology (3482/17 581). A day 1 positive test for DNA of HPV types $6,11,16$, and 18 was $4.1 \%$ (717/17 622), 0.7\% (120/17 622), 8.7\% (1553/17 622), and 3.6\% (641/17 622), respectively. Corresponding day 1 seropositivity was $8.2 \%$ (1438/ $17567), 2.0 \%$ (1036/17 566), 11.3\% (1980/17567), and $3.7 \%(646 / 17566)$, respectively.

Efficacy in preventing disease related to HPV types 6, 11, 16 , or 18

As expected, compared with the per protocol susceptible population (table 1), more cases of low grade cervical or vulvar intraepithelial neoplasia and condyloma were documented in the unrestricted susceptible and intention to treat populations (tables 2 and 3, respectively). Vaccine efficacy in the intention to 
Table 3|Efficacy of quadrivalent human papillomavirus (HPV) vaccine against low grade lesions attributable to vaccine HPV types (6, 11, 16, and 18$)$ : analysis of intention to treat population*

\begin{tabular}{|c|c|c|c|c|c|c|}
\hline \multirow[b]{2}{*}{ Lesion and related HPV type $†$} & \multicolumn{2}{|c|}{ Vaccine group } & \multicolumn{2}{|c|}{ Placebo group } & \multicolumn{2}{|c|}{ Vaccine efficacy $(\%(95 \% \mathrm{CI}))$} \\
\hline & $\begin{array}{l}\text { No of cases/ } \\
\text { No of subjects }\end{array}$ & $\begin{array}{c}\text { Person } \\
\text { years at risk }\end{array}$ & $\begin{array}{l}\text { No of cases/ } \\
\text { No of subjects }\end{array}$ & $\begin{array}{c}\text { Person } \\
\text { years at risk }\end{array}$ & Unadjusted & Adjusted $\ddagger$ \\
\hline Cervical intraepithelial neoplasia grade I (HPV 6, 11, 16, 18): & $114 / 8562$ & 29611.9 & $366 / 8598$ & 29473.1 & $69.0(61.6$ to 75.1$)$ & $68.8 \S(61.5$ to 74.7$)$ \\
\hline HPV 6 or 11 & $19 / 8562$ & 29688.6 & $87 / 8598$ & 29648.7 & $78.2(63.9$ to 87.5$)$ & $78.0 \S(63.9$ to 86.6$)$ \\
\hline HPV 16 & $81 / 8562$ & 29652.9 & $240 / 8598$ & 29601.6 & $66.3(56.5$ to 74.1$)$ & $66.0 \S(56.3$ to 73.6$)$ \\
\hline HPV 18 & $20 / 8562$ & 29701.7 & $91 / 8598$ & 29672.7 & $78.0(64.1$ to 87.2$)$ & 78.1 (64.4 to 86.5$)$ \\
\hline Vulvar intraepithelial neoplasia grade I (HPV 6, 11, 16, 18): & $8 / 8689$ & 30472.9 & $26 / 8702$ & 30563.7 & 69.1 (29.8 to 87.9$)$ & 69.1 (31.8 to 86.0$)$ \\
\hline HPV 6 or 11 & $8 / 8689$ & 30472.9 & $23 / 8702$ & 30570.7 & $65.1(19.2$ to 86.5$)$ & $65.1(22.0$ to 84.4$)$ \\
\hline HPV 16 & $0 / 8689$ & 30490.8 & $6 / 8702$ & 30602.0 & 100 ( $(0$ to 100$)$ & $100(\mathrm{NA})$ \\
\hline HPV 18 & $0 / 8689$ & 30488.1 & $0 / 8702$ & 30595.8 & NA & NA \\
\hline Vaginal intraepithelial neoplasia grade I (HPV 6, 11, 16, 18): & $4 / 8689$ & 30479.5 & $24 / 8702$ & 30568.6 & 83.3 (51.3 to 95.8$)$ & 83.3 (52.0 to 94.2$)$ \\
\hline HPV 6 or 11 & $2 / 8689$ & 30485.4 & $8 / 8702$ & 30599.2 & 74.9 ( 10 to 97.4$)$ & 75.0 ( 10 to 94.7$)$ \\
\hline HPV 16 & $2 / 8689$ & 30484.8 & $14 / 8702$ & 30590.1 & 85.7 (37.6 to 98.4) & 85.7 (37.1 to 96.8 ) \\
\hline HPV 18 & $1 / 8689$ & 30488.1 & $6 / 8702$ & 30595.8 & 83.3 ( 10 to 99.6$)$ & 83.3 ( 10 to 98.0$)$ \\
\hline Condyloma (HPV 6, 11, 16, 18): & $63 / 8689$ & 30326.2 & $305 / 8702$ & 30137.9 & 79.5 (73.0 to 84.6$)$ & $79.4 \S(73.0$ to 84.3$)$ \\
\hline HPV 6 or 11 & $62 / 8689$ & 30328.7 & $298 / 8702$ & 30151.2 & 79.3 (72.7 to 84.5$)$ & $79.2 \S(72.7$ to 84.2$)$ \\
\hline HPV 16 & $3 / 8689$ & 30481.8 & $32 / 8702$ & 30564.2 & 90.6 (70.0 to 98.2$)$ & 90.4 (68.6 to 97.1$)$ \\
\hline HPV 18 & $1 / 8689$ & 30487.1 & $22 / 8702$ & 30571.0 & 95.4 (71.8 to 99.9$)$ & 95.3 (65.1 to 99.4$)$ \\
\hline
\end{tabular}

*Intention to treat population=subjects who $(a)$ received $\geq 1$ vaccination; and $(b)$ had any follow-up visit. Case counting began after day 1 .

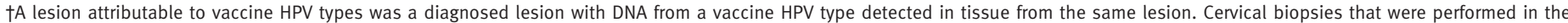
absence of an abnormal cervical smear test result at the antecedent visit were excluded.

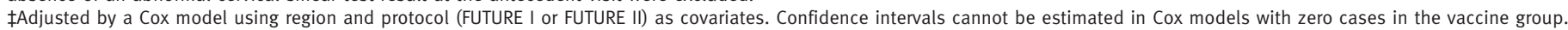

§Proportional hazard assumption was violated for the treatment effect. The vaccine efficacy was reported as average effect.

treat and unrestricted susceptible populations was generally lower than observed in the per protocol susceptible population, but it remained statistically significant for all four disease end points, ranging from $90 \%$ for vulvar intraepithelial neoplasia (2 cases in vaccine group $v 20$ cases in placebo group) to $100 \%$ for vaginal intraepithelial neoplasia ( $0 v 18$ cases) in the unrestricted susceptible population (table 2) and from 69\% for cervical intraepithelial neoplasia (114 v 366 cases) to $83 \%$ for vaginal intraepithelial neoplasia ( 4 v 24 cases) in the intention to treat population (table 3 ). Reductions were also seen in both populations when we evaluated vaccine efficacy against lesions attributable to specific vaccine HPV types (tables 2 and 3).

In the per protocol susceptible population (table 1), seven cases of cervical intraepithelial neoplasia attributable to HPV types $6,11,16$, or 18 were documented in the vaccine group $(n=7629)$ after month 7 compared with 168 cases in the placebo group $(n=7632)$ - translating into a vaccine efficacy of $96 \%$ (95\% confidence interval $91 \%$ to $98 \%$ ). Stratified by HPV type, vaccine efficacy rates against cervical intraepithelial neoplasia remained statistically significant and ranged from 94\% for HPV 16 to $100 \%$ for HPV 6 or 11 (table 1). With no cases of vulvar or vaginal intraepithelial neoplasia attributable to vaccine HPV types $(6,11,16$, or 18$)$ in the vaccine group compared with 16 cases and 12 cases, respectively, in the placebo group $(n=7669)$, vaccine efficacy was $100 \%$ in preventing these low grade neoplasias. All of the emergent vulvar cases in the placebo group were attributable to HPV types 6 or 11 , whereas all four vaccine HPV types were identified among the vaginal lesions. There were two cases of condyloma attributable to vaccine HPV types among vaccine recipients $(n=7665)$ versus 190 cases among placebo recipients ( $\mathrm{n}=7669)$ - a vaccine efficacy of $99 \%(96 \%$ to $100 \%)$. HPV type 6 was documented in both of the cases of condyloma in the vaccine group, and HPV types 6 or 11 were found in $98 \%$ of the placebo cases (186/190). Table 4 summarises the cases of lesions attributable to vaccine HPV types among vaccine recipients in the per protocol susceptible population.

\section{Efficacy in preventing disease due to any HPV type}

Vaccine efficacy against any cervical intraepithelial neoplasia grade I (regardless of HPV types present) was $30 \%(17 \%$ to $41 \%)$ in the generally HPV naive population (241 cases in vaccine group $v 346$ in placebo group) and $20 \%(12 \%$ to $28 \%)$ in the intention to treat population (788 v984 cases) (table 5). In the generally HPV naive population, vaccine efficacy against any vulvar intraepithelial neoplasia grade I, vaginal intraepithelial neoplasia grade I, or condyloma was $75 \%$ (22\% to $94 \%$ ) (4 v 16 cases), $48 \%$ (10\% to $71 \%$ ) (21 v 41 cases), and 83\% (74\% to 89\%) (29 v 169 cases) respectively (table 5 ). Corresponding efficacy in the intention to treat population was $32 \%(<0$ to $60 \%)(27$ $v 40$ cases), $31 \%$ (4\% to 51\%) (62 v 90 cases), and 62\% $(54 \%$ to $69 \%$ ) (134 v 351 cases), respectively (table 5 ).

\section{DISCUSSION}

We found that vaccination with quadrivalent HPV vaccine had a high prophylactic efficacy against low grade cervical and vulvovaginal neoplasias and condyloma attributed to HPV types 6, 11, 16, and 18 through 42 months of follow-up. This report confirms the 
Table 4|Description of cases of low grade lesions attributable to human papillomavirus (HPV) types $6,11,16$, or 18 among women in the per protocol susceptible population receiving quadrivalent HPV vaccine

\begin{tabular}{|c|c|c|c|c|c|}
\hline Case* $^{\star}$ & Age (years) & $\begin{array}{l}\text { Baseline HPV } \\
\text { positivity† }\end{array}$ & Detected & $\begin{array}{l}\text { Vaccine HPV } \\
\text { type found }\end{array}$ & Patient narrative \\
\hline \multicolumn{6}{|c|}{ Cervical intraepithelial neoplasia grade I } \\
\hline 1 & 17 & None & Month 13 & HPV 16 & $\begin{array}{l}\text { Colposcopy yielded two tissue specimens (both CIN grade I). Both biopsies were positive for HPV 58, one was } \\
\text { positive for HPV } 16 \text {. }\end{array}$ \\
\hline 2 & 20 & None & Month 13 & HPV 16 & Colposcopy yielded two tissue specimens, one of which was CIN grade I positive for HPV 16 \\
\hline 3 & 22 & $\begin{array}{l}\text { HPV } 18,31,33 \\
39\end{array}$ & Month 11 & HPV 16 & $\begin{array}{l}\text { Colposcopy yielded tissue specimen (read as CIN grade III) positive for HPV } 18,31,33 \text {, and 39. Definitive } \\
\text { therapy by LEEP yielded seven biopsies, only one of which was positive for HPV } 16 \text { (also positive for HPV } 18 \text {, } \\
\text { 33, and 39) and diagnosed as CIN grade I }\end{array}$ \\
\hline 4 & 23 & HPV 39 & Month 13 & HPV 16 & Colposcopy yielded two tissue specimens, one was normal and one was CIN grade I positive for HPV 16 \\
\hline 5 & 22 & HPV 56 & Month 35 & HPV 18 & $\begin{array}{l}\text { Colposcopy tissue specimen read as CIN grade II (positive for HPV 56, negative for HPV 18). Three LEEP } \\
\text { specimens were diagnosed as CIN grade I (all positive for HPV 56, and } 1 \text { positive for HPV 18) }\end{array}$ \\
\hline \multicolumn{6}{|c|}{ Condyloma } \\
\hline 6 & 18 & None & Month 8 & HPV 6 & \\
\hline 7 & 16 & None & Month 36 & HPV 6 & Condyloma positive for HPV 6 and 59 \\
\hline
\end{tabular}

results of previous studies with shorter follow-up times and increases the statistical power of the efficacy estimates (via longer follow-up) and provides evidence of longer duration of protection. There were no signs of waning protection.

\section{Putting these data in context}

The reported efficacy against low grade HPV related lesions is important for several reasons. Firstly, these lesions occur shortly after infection, and a reduction in these lesions will be the earliest clinically noticeable health gain to be realised by HPV vaccination. The incubation time for condyloma is on average about three months, and a decline in these readily apparent lesions is an early monitoring end point for confirming that HPV vaccination programmes have had an important effect at the population level. Monitoring for outbreaks with re-emergence of condylomas has been proposed as an integral part of HPV surveillance programmes after vaccination.

Secondly, condyloma and cervical intraepithelial neoplasia grade I occur at substantially higher rates than cervical intraepithelial neoplasia grade II/III, and the absolute number of these cases prevented by vaccination is expected to be large when vaccine coverage is high. Thus, short term benefits of HPV vaccination in sexually active populations lie in the reduction of condylomas and cervical intraepithelial neoplasia grade I.

Thirdly, the fact that infection with multiple HPV types is common in low grade disease, particularly in cervical intraepithelial neoplasia but also in condyloma, has made it difficult to unequivocally assign which of the HPV types present in a lesion are the causal infections. Our data therefore provide important confirmatory evidence of the proportion of low grade disease positive for HPV types $6,11,16$, or $18(37 \%$ (366/984) of cervical intraepithelial neoplasia grade I, $87 \%(305 / 351)$ of condyloma (tables 3 and 5 , intention to treat placebo groups)). As expected, the proportion of disease prevented in generally naive subjects was similar but slightly lower than the proportion of cases found to contain the DNA of these viruses (30\% of cervical intraepithelial neoplasia grade I, 83\% of condyloma). Co-infection with a non-vaccine HPV type can result in uncertainty in assigning causality, as shown by the substantial proportion of the cases of disease in the vaccine group that were also positive for a non-vaccine HPV type (3/7 cases of cervical intraepithelial neoplasia grade I and 1/2 cases of condyloma in the per protocol population). In several cases the additional HPV type had been detected at the baseline visit and persisted through the study.

Low grade cervical and vulvovaginal lesions are important from a public health perspective, as the diagnosis, follow-up, and treatment of these common lesions are associated with substantial patient anxiety, morbidity, and healthcare costs. ${ }^{1024}$ The lifetime risk of a clinically diagnosed condyloma has in Scandinavia been estimated to be $>10 \% .{ }^{25}$ Management of cervical intraepithelial neoplasia grade I and condyloma therefore contributes to a large proportion of the overall financial burden of HPV related disease. ${ }^{26}$

\section{Condyloma and HPV vaccination}

The incidence of condyloma and the potential for health gains by HPV vaccination has hitherto not been well described. Although condyloma reporting systems are used in a few countries, these systems have not been sufficiently well controlled to allow reliable estimates of incidence. In this study we measured vaccine efficacy against disease related to specific HPV type and against disease regardless of HPV type, enabling an estimate of the total gains in reduced disease burden after vaccination of generally HPV naive women. In addition, our report provides an estimate of the incidence of these lesions in a carefully monitored cohort of women. We found a high incidence of condyloma in the placebo group (169 cases), which translates into a yearly incidence of $1.0 \%$. The vaccine efficacy against condyloma 
Table 5 | Efficacy of quadrivalent human papillomavirus (HPV) vaccine against low grade lesions attributable to any HPV type

\begin{tabular}{|c|c|c|c|c|}
\hline & \multicolumn{2}{|c|}{ No of cases/No of subjects } & \multicolumn{2}{|c|}{ Vaccine efficacy $(\%(95 \% \mathrm{Cl}))$} \\
\hline & Vaccine group & Placebo group & Unadjusted & Adjusted* \\
\hline \multicolumn{5}{|l|}{ Generally HPV naive population $†$} \\
\hline Cervical intraepithelial neoplasia grade I & $241 / 4616$ & $346 / 4680$ & 29.7 (16.9 to 40.6) & $29.9(17.3$ to 40.5$)$ \\
\hline Vulvar intraepithelial neoplasia grade I & $4 / 4689$ & $16 / 4735$ & 74.7 (21.5 to 93.8$)$ & $74.6(23.9$ to 91.5$)$ \\
\hline Vaginal intraepithelial neoplasia grade I & $21 / 4689$ & $41 / 4735$ & 48.1 (10.2 to 70.9$)$ & $49.2(14.0$ to 70.0$)$ \\
\hline Condyloma & $29 / 4689$ & $169 / 4735$ & 82.8 (74.3 to 88.8$)$ & $82.8(74.5$ to 88.4$)$ \\
\hline \multicolumn{5}{|l|}{ Intention to treat population $\ddagger$} \\
\hline Cervical intraepithelial neoplasia grade I & $788 / 8562$ & $984 / 8598$ & 20.3 (12.4 to 27.5$)$ & 20.0 (12.1 to 27.2$)$ \\
\hline Vulvar intraepithelial neoplasia grade I & $27 / 8689$ & $40 / 8702$ & 32.3 ( $<0$ to 60.0$)$ & 32.1 ( $<0$ to 58.4$)$ \\
\hline Vaginal intraepithelial neoplasia grade I & $62 / 8689$ & $90 / 8702$ & 30.9 (3.5 to 50.8$)$ & $31.2(4.9$ to 50.2$)$ \\
\hline Condyloma & $134 / 8689$ & $351 / 8702$ & $62.0(53.5$ to 69.1$)$ & $61.9 \S(53.5$ to 68.8$)$ \\
\hline
\end{tabular}

*Vaccine efficacy was adjusted by a Cox model using region and protocol (FUTURE I and FUTURE II) as covariates.

†Generally HPV naive population=subjects who (a) received $\geq 1$ vaccination; (b) tested negative at day 1 for vaccine HPV types $(6,11,16$, and 18$)$ by serology and polymerase chain reaction (PCR) and for non-vaccine, high risk HPV types $(31,33,35,39,45,51,52,56,58$, and 59$)$ by PCR and had a negative cervical smear test on day 1 ; and $(c)$ had any follow-up visit. Case counting began after day 1.

fIntention to treat population=subjects who $(a)$ received $\geq 1$ vaccination; and (b) had any follow-up visit. Case counting began after day 1.

§Proportional hazard assumption was violated for the treatment effect. The vaccine efficacy was reported as average effect.

in generally HPV naive women of $83 \%$ thus corresponds to a potential reduction in absolute yearly incidence of condyloma of $0.83 \%$.

In accordance with previous reports, we found oncogenic HPV types such as HPV 16 and 18 in a proportion of condylomas, but usually in conjunction with the major HPV types associated with condyloma (types 6 and 11). Of the 190 cases of condyloma in the per protocol placebo group, 23 were associated with HPV type 16 , and 11 associated with HPV type 18 . However, HPV types 6 or 11, or both, were also present in all but four of these. Therefore, it seems likely that the quadrivalent vaccine's efficacy against condyloma is primarily attributable to the HPV types 6 and 11 components of the vaccine.

For cervical intraepithelial neoplasia grade I, HPV type 16 was found in about twice as many cases (97/ 6257) as HPV type 18 (47/7092) and types 6 or 11 (45/ 6619). As multiple infection with several vaccine types was not common, it seems reasonable to assume that the vaccine's HPV types 6 and 11 component contributed to about a quarter of the protective effect - that is, about 7\%-8\% of all cervical intraepithelial neoplasia grade I. Thus, the proportion of disease preventable by vaccination against HPV types 6 and 11 was slightly lower than the prevalence of types 6 or 11 in low grade cervical and vulvovaginal disease (estimated at about $10 \%$ for cervical intraepithelial neoplasia grade I, 42\% for vulvar intraepithelial neoplasia grade I, and 90\% for condyloma ${ }^{10122728}$ ). This observation may possibly be related to the occasional cases that may be caused by co-infection with non-vaccine HPV types.

For vulvar intraepithelial neoplasia grade I, our data support the International Society for the Study of Vulvovaginal Disease recommendation to rename this as a "flat wart," as all cases of vulvar intraepithelial neoplasia grade I were found to harbour HPV types 6 or 11 and thus had similar aetiology as other condylomas. For vaginal intraepithelial neoplasia grade I, about equal numbers of cases were infected with HPV types 6 or 11 as with HPV 16, but small numbers preclude more exact estimation of the proportion of the protective effect against vaginal intraepithelial neoplasia that was attributable to the HPV 6 and 11 component of the vaccine. Regardless of whether vulvar or vaginal intraepithelial neoplasia grade I are classified as precancerous or as benign warts, patients presenting with low grade vulvar or vaginal lesions still require clinical follow-up and, in some cases, medical or surgical intervention.

\section{Study limitations}

Limitations of the current analyses include the fact that the generally HPV naive population was tested only for the presence of the four vaccine HPV types and 10 other HPV types prevalent in cervical cancer. However, other HPV types may contribute to condylomas, and there are several uncommon HPV types that we did not test for (such as HPV 68 and 73) that are classified as oncogenic. Failure to identify infections with these types at baseline would have resulted in an underestimation of the protective effect against any disease in the generally naive population. As these types are not common, this conservative bias is not likely to be substantial. Another limitation concerns the fact that rate estimates of disease are dependent on the intensity of assessment. There were some differences in assessment between studies, notably the fact that protocol 015 required cervical smear screening every 12 months, whereas protocol 013 required smear testing every six months. Although the studies eligibility criteria included a limit on the lifetime number of sexual partners, the generalisability of the results is probably high as the population studied was enrolled globally.

\section{Conclusions and policy implications}

Quadrivalent HPV vaccination provided strong and sustained protection for up to four years against condyloma and low grade cervical and vulvovaginal neoplasia related to HPV types $6,11,16$, and 18 . The high incidence of low grade disease seen in the placebo group and the estimated benefits of vaccination on total disease burden regardless of HPV type suggest 


\section{WHAT IS ALREADY KNOWN ON THIS TOPIC?}

Human papillomaviruses (HPVs) are responsible for substantially more cases of condyloma and cervical intraepithelial neoplasia grade I than cervical intraepithelial neoplasia grade IIIII, and the absolute number of these cases prevented by HPV vaccination is expected to be large

The total disease burden of low grade lesions that is preventable by quadrivalent HPV vaccine has not been well elucidated

\section{WHAT DOES THIS STUDY ADD?}

Quadrivalent vaccine (for HPV types 6,11,16, and 18) provides strong and sustained protection for up to four years against condyloma and low grade cervical and vulvovaginal neoplasia related to these four HPV types

In generally HPV naive subjects, vaccination reduced the total burden of condyloma by $83 \%$ and the total burden of cervical intraepithelial neoplasia grade I by $30 \%$

The HPV types 6 and 11 component of the vaccine contributed to about a quarter of the protective effect against cervical intraepithelial neoplasia grade I related to any HPV type in the intention to treat population
Obstetrics and Gynecology, Duke University Medical Center, Durham, NC, USA; ${ }^{25}$ Department of Virology, Ludwig Institute for Cancer Research, Sao Paulo, Brazil; ${ }^{26}$ Merck Research Laboratories, West Point, PA, USA Contributors: $E B, R H$, and TMH managed the sponsor's operations. JD, CMW, KS, O-EI, MH-A, DRB, LAK, EHT, PG, KAA, SMG, SLe, S-EO, GWKT, DGF, JP, ML, MS, EAJ, GP, and SM set up study sites and enrolled participants into the studies. SKK, GP, NM, FXB, ERM, and LLV helped draft the protocols. JTB, FJT, CR, and AT managed the development of the PCR based assays for HPV types 6, 11, 16, and 18 as well as the execution of genital swab and biopsy sample testing using the assays. RM and SLu provided statistical expertise. JD and SV drafted the manuscript, to which all others contributed and approved before submission.

Funding: Merck Research Laboratories, a division of Merck \& Company, funded these studies.

Role of the funding source: The studies were designed by the sponsor (Merck \& Company) in collaboration with external investigators and an external data and safety monitoring board. The sponsor collated the data, monitored the conduct of the study, performed the statistical analysis, and coordinated the writing of the manuscript with all authors. The authors were actively involved in the collection, analysis, or interpretation of the data, the revising of the manuscript for intellectual content, and approved the final manuscript.

Competing interests: JD has received consultancy fees, lecture fees, and research grants from Merck \& Company and Sanofi Pasteur MSD. SKK has received consultancy fees and has received funding through her institution to conduct HPV vaccine studies for Sanofi Pasteur MSD and Digene. SMG has received advisory board fees and grant support from Commonwealth Serum Laboratories and GlaxoSmithKline, lecture fees from Merck \& Company, and funding through her institution to conduct HPV vaccine studies for GlaxoSmithKline. CMW has received funding through her institution to conduct HPV vaccine studies for

GlaxoSmithKline, and has received reagents and equipment from Roche Molecular Systems for HPV genotyping studies. KS has received consultancy fees from Merck \& Company. O-El has received lecture fees from Merck \& Company and GlaxoSmithKline. LLV has received lecture fees, advisory board fees, and consultancy fees from Merck \& Company and Sanofi Pasteur MSD. KAA has received consultancy and advisory board fees. MH-A has received lecture fees and grant support from Merck \& Company. GP has received lecture fees and consultancy fees from Merck \& Company and Sanofi Pasteur MSD. DRB has received lecture fees, advisory board fees, and intellectual property fees. SLe has received lecture fees from Merck \& Company and Sanofi Pasteur MSD. S-EO has received lecture fees from Merck \& Company. DGF has received consultancy fees and funding through his institution to conduct HPV vaccine studies for GlaxoSmithKline, and lecture fees and consultancy fees from Merck \& Company. JP has received consultancy fees, advisory board fees, and lecture fees from Merck \& Company. MS has received lecture fees and grant support from Merck \& Company. FXB has received lecture fees from Merck \& Company and GlaxoSmithKline, and has received funding through his institution to conduct HPV vaccine studies for GlaxoSmithKline. EAJ has received lecture fees from Merck \& Company, Sanofi Pasteur MSD, and GlaxoSmithKline. SM has received lecture fees, advisory board fees, and consultancy fees from Merck \& Company and Sanofi Pasteur MSD. NM has received lecture fees, advisory board fees, and consultancy fees from Merck \& Company and Sanofi Pasteur MSD. Additionally, S-EO, CMW, MH-A, LLV, O-EI, GWKT, FXB, JP, JD, EHT, SLe, EAJ, SKK, GP, DGF, KS, MS, LAK, and DRB have received funding through their institutions to conduct HPV vaccine studies for Merck \& Company. FJT, CR, AT, JTB, RM, SV, TMH, RH, and EB are employees of Merck \& Company and potentially own stock or stock options in the company.

Ethical approval: Studies were conducted in conformity with country or local requirements regarding ethics committee review, informed consent, and other statutes or regulations regarding the rights and welfare of human subjects participating in biomedical research.

Data sharing: Data sets are available from the corresponding author at joakim.dillner@med.lu.se.

1 Ferlay J, Bray F, Pisani P, Parkin DM. GLOBOCAN 2002: cancer incidence, mortality and prevalence worldwide. IARC CancerBase No 5, version 2.0. IARCPress, 2004

2 World Health Organization. Human papillomavirus infection and cervical cancer.

2004. www.who.int/vaccine_research/diseases/hpv/en/. National de Santé Publique du Québec, Montréal, Canada; ${ }^{21}$ Institut Catala d'Oncologia, IDIBELL, Barcelona, Spain; ${ }^{22}$ Department of Dermatology and Venereology, Center of Diagnostics and Treatment of Sexually Transmitted Diseases, Warsaw Medical University, Warsaw, Poland; ${ }^{23}$ National Institute of Cancer, Bogotá, Colombia; ${ }^{24}$ Department of 
3 Clarke P, Ebel C, Catotti DN, Stewart S. The psychosocial impact of human papillomavirus infection: implications for health care providers. Int J STD AIDS 1996;7:197-200.

4 Maw RD, Reitano M, Roy M. An international survey of patients with genital warts: perceptions regarding treatment and impact on lifestyle. Int J STD AIDS 1998;9:571-8.

5 Insinga RP, Dasbach EJ, Myers ER. The health and economic burden of genital warts in a set of private health plans in the United States. Clin Infect Dis 2003;36:1397-403.

6 Langley PC, White DJ, Drake SM. The costs of treating external genita warts in England and Wales: a treatment pattern analysis. Int J STD AIDS 2004;15:501-8.

7 Kodner CM, Nasraty S. Management of genital warts. Am Fam Physician 2004;70:2335-42.

8 Lacey CJ. Therapy for genital human papillomavirus-related disease.) Clin Virol 2005;32:S82-S90.

9 Wiley DJ, Douglas J, Beutner K, Cox T, Fife K, Moscicki AB, et al. External genital warts: diagnosis, treatment and prevention. Clin Infect Dis 2002;35:S210-S224.

10 Clifford GM, Rana RK, Franceschi S, Smith JS, Gough G, Pimenta JM Human papillomavirus genotype distribution in low-grade cervical lesions: comparison by geographic region and with cervical cancer. Cancer Epidemiol Biomarkers Prev 2005;14:1157-64.

11 Herrero R, Castle PE, Schiffman M, Bratti MC, Hildesheim A, Morales J, et al. Epidemiologic profile of type-specific human papillomavirus infection and cervical neoplasia in Guanacaste, Costa Rica. J Infect Dis 2005;191:1796-807.

12 Srodon M, Stoler MH, Baber GB, Kurman RJ. The distribution of low and high-risk HPV types in vulvar and vaginal intraepithelial neoplasia (VIN and VaIN). Am J Surg Pathol 2006;30:1513-8.

13 Von Krogh G. Management of anogenital warts (condylomata acuminata). Eur J Dermatol 2001;11:598-604.

14 Garland SM, Steben M, Sings HL, James M, Lu S, Railkar R, et al. Natural history of genital warts: analysis of the placebo arm of 2 randomized phase III trials of a quadrivalent human papillomavirus (types 6, 11, 16, and 18) vaccine. I Infect Dis 2009;199:805-14.

15 Muñoz N, Bosch FX, de Sanjosé S, Herrero R, Castellsaguë X, Shah KV, et al. Epidemiologic classification of human papillomavirus types associated with cervical cancer. $N$ Engl J Med 2003;348:518-27.

16 The FUTURE II Study Group. Effect of prophylactic human papillomavirus L1 virus-like-particle vaccine on risk of cervical intraepithelial neoplasia grade 2 , grade 3 and adenocarcinoma in situ: a combined analysis of four randomised clinical trials. Lancet 2007;369:1861-8

17 Joura EA, Leodolter S, Hernandez-Avila M, Wheeler CM, Perez G, Koutsky LA, et al. Efficacy of a quadrivalent prophylactic human papillomavirus (types 6,11,16 and 18) L1 virus-like-particle vaccine against high-grade vulval and vaginal lesions: a combined analysis of three clinical trials. Lancet 2007;369:1693-702.

18 Garland SM, Hernandez-Avila M, Wheeler CM, Perez G, Harper DM, Leodolter S, et al. Quadrivalent vaccine against human papillomavirus to prevent anogenital diseases. N Engl / Med 2007;356:1928-43.

19 The FUTURE II Study Group. Quadrivalent vaccine against human papillomavirus to prevent high-grade cervical lesions. N Engl J Med 2007;356:1915-27.

20 Solomon D, Davey D, Kurman R, Moriarty A, O'Connor D, Prey M, et al. The 2001 Bethesda system: terminology for reporting results of cervical cytology. JAMA 2002;287:2114-9.

21 Sideri M, Jones RW, Wilkinson EJ, Preti M, Heller DS, Scurry J, et al. Squamous vulvar intraepithelial neoplasia: 2004 modified terminology, ISSVD Vulvar Oncology Subcommittee. J Reprod Med 2005;50:807-10.

22 Chan ISF, Bohidar NR. Exact power and sample size for vaccine efficacy studies. Theory Meth 1998;27:1305-22.

23 The FUTURE II Study Group. Prophylactic efficacy of a quadrivalent human papillomavirus (HPV) vaccine in women with virologic evidence of HPV infection. J Infect Dis 2007;196:1438-46.

24 Insinga RP, Glass AG, Rush BB. The health care costs of cervical human papillomavirus-related disease. Am J Obstet Gynecol 2004;191:114-20.

25 Kjaer SK, Tran TN, Sparen P, Tryggvadottir L, Munk C, Dasbach E, et al. The burden of genital warts: a study of nearly 70,000 women from the general female population in the 4 Nordic countries. I Infect Dis 2007;196:1447-54

26 Bergeron C, Breugelmans JG, Bouee S, Lorans C, Benard S, Remy V. [Cervical cancer screening and associated treatment costs in France.] Gynecol Obstet Fertil 2006;34:1036-42.

27 Jansen KU, Shaw AR. Human papillomavirus vaccines and prevention of cervical cancer. Annu Rev Med 2004;55:319-31.

28 Greer CE, Wheeler CM, Ladner MB, Beutner K, Coyne MY, Liang H, et al. Human papillomavirus (HPV) type distribution and serological response to HPV type 6 virus-like particles in patients with genital warts. J Clin Microbiol 1995;33:2058-63.

Accepted: 29 April 2010 